\title{
El mercado laboral de los titulados en Biblioteconomía y Documentación: análisis de las ofertas de empleo publicadas en IWETEL durante el periodo 2000-2007
}

\author{
Carlos Hugo Artaza \\ Universidad Nacional de Mar del Plata. Facultad de Humanidades \\ Departamento de Documentación - Argentina
}

ARTÍCULO / ARTICLE

\begin{abstract}
Resumen
Con la finalidad de describir las características que presentan las ofertas laborales destinadas a titulados en Biblioteconomía y Documentación en España se analizaron los ofrecimientos publicados en la lista de interés IWETEL durante el periodo 2000 2007. Con las ofertas se efectuaron análisis estadísticos referidos a: número de empleos ofertados, tipología de las organizaciones demandantes, requisitos solicitados y modalidad de los contratos. Se concluye que la mayor demanda de empleos proviene de organizaciones empresariales. Las organizaciones demandantes son mayormente del ámbito privado y el tipo de contrato ofrecido de carácter temporario. En relación a lo conocimientos requeridos, las áreas de Tecnología de la Información, Organización y Tratamiento de la Información e Idiomas figuran entre los más demandados por los empleadores.
\end{abstract}

Palabras clave

Mercado de trabajo ; Biblioteconomía ; Documentación ; Bibliotecarios ; Documentalistas ; Listas de interés ; IWETEL

The job market for graduates in library and information science: analysis of job advertisements published in IWETEL during the period 2000-2007

\begin{abstract}
In order to determine the characteristics that have the job offers for graduates in Library and Information Science in Spain were analyzed the jobs advertised in IWETEL during the period 2000-2007. With offers were performed statistical analysis in relation to: number of jobs offered, typology of organizations applying, qualification requirements and type of contracts. We conclude that the largest number of orders of jobs comes from business organizations. The applicants organizations are mostly of the private sector and the type of contract offer of a temporary nature. In relation to the knowledge required, the areas of Information Technology, Organization and Information Processing and Language, are among the most demanded by employers.
\end{abstract}

Keywords

Labour market ; Library and information science ; Librarians ; Documentalists ; Interest lists ; IWETEL 


\section{Introducción}

En distintos países la inserción laboral de los titulados en Biblioteconomía y Documentación ha sido objeto de numerosas investigaciones. En general el objeto de estos trabajos ha sido investigar en profundidad qué tipo de organizaciones requieren de profesionales, cuales son las habilidades y conocimientos solicitados y qué características presentan los empleos ofertados. En España se han realizado varios estudios referidos a esta temática. Seguidamente repaceremos los principales aportes realizados.

Espelt y Pons (1993) efectuaron una de las primeras investigaciones referidas a la temática que nos ocupa. Los autores analizaron la inserción laboral de los egresados de la Escuela de Biblioteconomía Jordi Rubio i Balaguer de Barcelona entre los años 1988 y 1992. Para realizar su investigación tomaron una muestra de los graduados registrados en el Collegi Oficial de Bibliotecaris Documentalistes de Catalunya. En el estudio se concluye que la mayor parte de los profesionales trabajaba en el sector público y un porcentaje mínimo (5,6\%) lo hacía en empresas privadas.

Por su parte Moreiro, Moscoso y Ortiz-Repiso (1995) realizaron un estudio con los egresados de la promoción de 1992 de cuatro escuelas de biblioteconomía (Barcelona, Granada, Murcia y Salamanca). El estudio arrojó como resultado que el $79 \%$ de los diplomados trabajaban en organismos públicos: en bibliotecas (69\%) y en centros de documentación (17\%).

Camps y Crespán (1996), analizaron también una muestra de miembros del Col-legi Oficial de BibliotecarisDocumentalistes de Catalunya concluyendo que casi tres cuartas partes de los titulados tuvieron su primer empleo a través de organismos e instituciones públicas.

Muñoz Escolá (1997) efectuó posteriormente un estudio de los egresados de la Universidad de Zaragoza. El autor analizó la inserción laboral en las cuatro primeras promociones de la diplomatura. El estudio arrojo como resultado que solo el $36 \%$ de los egresados trabajaba y de ese porcentaje un $21 \%$ lo hacía en lugares no relacionados con sus estudios. El $79 \%$ restante que se desempeñaba en tareas relacionadas con su formación lo hacía mayoritariamente en bibliotecas (52\%) y centros de documentación (30\%).

Durante el período 1995-96 Moreiro y Caridad (1998) estudiaron las convocatorias para estudiantes de la diplomatura en Biblioteconomía y Documentación en la bolsa de empleo del Servicio de Orientación y Planificación Profesional de la Fundación Universidad Carlos III de Madrid. En su mayoría los pedidos provenían del ámbito de la Administración pública (17 ofertas para 46 puestos), consultoría (11 ofertas para 14 puestos) y servicios a empresas (9 ofertas para 10 puestos).

Galán Cruzado (1999) por su parte estudió durante dos años las convocatorias de oposiciones en Archivística, Biblioteconomía, Documentación y Museología publicadas en el Boletín oficial del Estado y el Boletín oficial de la Junta de Andalucía. Los resultados muestran un total de 50 convocatorias y de 96 plazas. Las universidades ofrecieron el mayor número de puestos de trabajo (47 plazas), seguidas de los ayuntamientos (39).

Alonso y Vázquez (2000) efectuaron un análisis de las ofertas laborales publicadas en la lista de correo electrónico InfoDOC. En su mayoría las ofertas provenían del sector público: bibliotecas ( $55 \%$ de las plazas), servicios de documentación (21\%) y archivos (13\%).

Frías y otros autores (2000) investigaron sobre la inserción laboral de los diplomados en Biblioteconomía y Documentación y los licenciados en Documentación egresados de la Universidad de Salamanca. Los autores analizaron las ofertas de empleo publicadas en el Boletín Oficial de Castilla y León durante el periodo comprendido entre los años 1991 y 1998. Los resultados del estudio señalaron que el mayor número de solicitudes correspondía a puestos para bibliotecas ( $80 \%$ de las plazas) y en menor proporción a trabajos en archivos (11\%).

Sanz-Casado, Martin-Moreno, García-Zorita, Lascurian-Sanchez (2002) también realizaron una investigación para establecer la relevancia de las temáticas de los estudios de biblioteconomía en las universidades en España en relación con los empleos buscados por los estudiantes. En el estudio se destaca que en los requerimientos profesionales presentes en las ofertas laborales, se solicitan frecuentemente conocimientos referidos a catalogación, clasificación, manejo y la elaboración de tesauros, manejo de nuevas tecnologías y de herramientas de gestión documental, técnicas de gestión de información y conocimiento de idiomas, predominantemente el inglés. 
En otro estudio realizado por la Universidad de Barcelona (2003) sobre la inserción laboral de los graduados en Biblioteconomía y Documentación entre los años 1998 y 2003, se comprobó que las bibliotecas, principalmente públicas, constituyen la principal fuente de empleo para los egresados. En la investigación también se observó que un $33,1 \%$ de los egresados poseía un contrato de trabajo permanente, un $29,4 \%$ un contrato laboral temporal y un $17,5 \%$ eran funcionarios. Con respecto a la inserción de los licenciados los resultados son similares y en relación al nivel de los empleos, el $47 \%$ correspondían a funcionarios, un $23,5 \%$ a contratos laborales permanentes y un $12 \%$ a contratos laborales temporales.

En los estudios que hemos analizado hemos observado como los autores han tratado de constatar cuales son las características principales que presentan las solicitudes laborales a los egresados de las carreras de documentación. Estas investigaciones han permitido mejorar nuestro conocimiento sobre los perfiles profesionales y las competencias que son requeridas por la sociedad. También han proporcionado información de utilidad respecto a los contenidos que deberían priorizarse en los diseños curriculares de las carreras para mejorar la inserción laboral de los egresados.

En nuestro estudio también nos hemos ocupado de analizar las demandas laborales destinadas a los diplomados de bibliotecario y de licenciado en biblioteconomía. Para ello hemos realizado una investigación de las ofertas de trabajo publicadas en la lista de interés IWETEL. El objeto del trabajo ha sido describir las principales características que presentan las solicitudes laborales (número de empleos ofertados, tipología de las organizaciones demandantes, requisitos solicitados y modalidad de los contratos) durante el periodo 2000- 2007.

\section{Materiales y métodos}

La investigación de carácter descriptiva, longitudinal y retrospectiva se realizó utilizando las ofertas de trabajo destinadas a bibliotecarios y licenciados publicadas en la lista IWETEL, principal foro electrónico sobre bibliotecas y documentación de España.

Para obtener la información se consultaron los archivos de e-mail disponibles en el sitio Web de la lista. El estudio abarcó el periodo comprendido entre los años 2000 y 2007. Por cada año se analizaron las ofertas de pedidos de bibliotecarios y de licenciados en biblioteconomía. Las ofertas de empleo destinadas a estudiantes de las carreras no fueron incluidas. Con la información relevada se conformó una base de datos en MS-Access. Los datos fueron relacionados y procesados estadísticamente a fin de comparar y analizar los requerimientos profesionales demandados.

Se efectuaron análisis referidos a los siguientes ítems:

- Cantidad de empleos ofertados.

- Tipos de organizaciones que realizan ofertas.

- Conocimientos requeridos.

- Ámbito de las organizaciones y modalidades de los contratos.

\section{Resultados}

A través de la investigación pudimos caracterizar las ofertas laborales destinadas a bibliotecarios y licenciados en biblioteconomía, publicadas en la lista de interés IWETEL. Seguidamente se presentan los principales resultados obtenidos.

\section{Cantidad de empleos ofertados}

Se analizaron un total de 595 solicitudes de empleo. Se presentaron ofertas tanto para bibliotecarios como para licenciados en biblioteconomía. Del total de solicitudes el mayor número de pedidos correspondió a las ofertas para bibliotecarios con 427 solicitudes $(71,76 \%)$ y el menor número a licenciados con 168 ofrecimientos $(28,23 \%)$. 


\section{Tipos de organizaciones que realizan ofertas}

Las organizaciones que ofrecen puestos de trabajo presentan variantes en su tipología. En las ofertas para bibliotecarios, sobre un total de 427 ofrecimientos, el 52,45\% correspondió a empresas, el $29,50 \%$ a bibliotecas, el $13,58 \%$ a centros de documentación, el 3,98\% a archivos y el $0,46 \%$ a librerías. Con respecto a las ofertas de las empresas hay que destacar que el 33,72\% correspondió a empresas de servicios documentales (procesamiento técnico de materiales y desarrollo bases de datos mayormente) y el 11,47\% a empresas consultoras. En relación a las bibliotecas las mayores ofertas provienen de las bibliotecas públicas $(10,30 \%)$ y universitarias $(9,13 \%)$. Las ofertas para licenciados presentan características similares. Sobre un total de 168 ofrecimientos el 66,66\% correspondió a empresas, el 13,69\% a bibliotecas, el 10,71\% a archivos y el 8,92\% a centros de documentación. Con respecto a las empresas, al igual que en las ofertas para bibliotecarios, el mayor porcentaje correspondió a empresa de servicios documentales (51,78\%). En el caso de las bibliotecas el mayor número de ofertas correspondió a bibliotecas especializadas $(5,95 \%)$ y universitarias $(4,76 \%)$. Es de destacar también la importante presencia de ofertas laborales para licenciados en distintos tipos de archivos históricos (7,73\%). En las siguientes tablas se presentan en detalles los diferentes tipos de organizaciones que ofrecen puestos de trabajo:

\begin{tabular}{|l|c|c|c|c|}
\hline \multicolumn{1}{|c|}{ IWETEL } & \multicolumn{2}{|c|}{ BIBLIOTECARIO } & \multicolumn{2}{c|}{ LICENCIADO } \\
\hline \multicolumn{1}{|c|}{ TIPO DE ORGANIZACIÓN } & CANTIDAD & PORCENTAJE & CANTIDAD & PORCENTAJE \\
\hline ARCHIVOS & 17 & 3,98 & 18 & 10,71 \\
\hline BIBLIOTECAS & 126 & 29,50 & 23 & 13,69 \\
\hline CENTROS DE DOCUMENTACIÓN & 58 & 13,58 & 15 & 8,92 \\
\hline EMPRESAS & 224 & 52,45 & 112 & 66,66 \\
\hline LIBRERIAS & 2 & 0,46 & 0 & 0,00 \\
\hline TOTALES & 427 & 100 & 168 & 100 \\
\hline
\end{tabular}

Tabla 1 - Resumen de los Tipos de Organización que ofertan empleos.

\begin{tabular}{|l|c|c|c|c|}
\hline \multicolumn{1}{|c|}{ IWETEL } & \multicolumn{2}{c|}{ BIBLIOTECARIO } & \multicolumn{2}{c|}{ LICENCIADO } \\
\hline \multicolumn{1}{|c|}{ TIPO DE ORGANIZACIÓN } & CANTIDAD & PORCENTAJE & CANTIDAD & PORCENTAJE \\
\hline ARCHIVO BANCO & 1 & 0,23 & 0 & 0,00 \\
\hline ARCHIVO EMPRESA & 2 & 0,46 & 3 & 1,78 \\
\hline ARCHIVO FOTOGRAFICO & 5 & 1,17 & 0 & 0,00 \\
\hline ARCHIVO HISTÓRICO & 8 & 1,87 & 13 & 7,73 \\
\hline ARCHIVO JURÍDICO & 0 & 0,00 & 2 & 1,19 \\
\hline ARCHIVO LABORATORIO & 1 & 0,23 & 0 & 0,00 \\
\hline BIB. NACIONAL & 4 & 0,93 & 1 & 0,59 \\
\hline BIB. ADMINISTRACIÓN PÚBLICA & 2 & 0,46 & 0 & 0,00 \\
\hline BIB. DE MUSEO & 3 & 0,70 & 0 & 0,00 \\
\hline BIB. ESCOLAR PRIMARIA & 7 & 0,00 & 1,63 & 4 \\
\hline BIB. ESPECIALIZADA ARQUITECTURA & & & 2,38 \\
\hline
\end{tabular}




\begin{tabular}{|c|c|c|c|c|}
\hline BIB. ESPECIALIZADA ADMINISTRACIÓN EMPRESAS & 2 & 0,46 & 0 & 0,00 \\
\hline BIB. ESPECIALIZADA ARTE & 15 & 3,50 & 6 & 3,57 \\
\hline BIB. ESPECIALIZADA CIENCIAS SOCIALES & 2 & 0,46 & 1 & 0,59 \\
\hline BIB. ESPECIALIZADA HOTELERÍA & 1 & 0,23 & 0 & 0,00 \\
\hline BIB. ESPECIALIZADA LITERATURA & 5 & 1,16 & 0 & 0,00 \\
\hline BIB. JURÍDICA & 1 & 0,23 & 0 & 0,00 \\
\hline BIB. PÚBLICA & 44 & 10,30 & 2 & 1,19 \\
\hline BIB. UNIVERSITARIA & 39 & 9,13 & 8 & 4,76 \\
\hline CENTRO DE DOCUMENTACIÓN CIENCIA Y TECNOLOGÍA & 5 & 1,17 & 4 & 2,38 \\
\hline CENTRO DE DOCUMENTACIÓN CIENCIAS NATURALES & 1 & 0,23 & 0 & 0,00 \\
\hline CENTRO DE DOCUMENTACIÓN CIENCIAS POLÍTICAS & 1 & 0,23 & 0 & 0,00 \\
\hline CENTRO DE DOCUMENTACIÓN CIENCIAS SOCIALES & 4 & 0,93 & 0 & 0,00 \\
\hline CENTRO DE DOCUMENTACIÓN DEPORTES & 1 & 0,23 & 0 & 0,00 \\
\hline CENTRO DE DOCUMENTACIÓN EDUCACIÓN & 3 & 0,70 & 0 & 0,00 \\
\hline CENTRO DE DOCUMENTACIÓN EMPRESA & 2 & 0,46 & 0 & 0,00 \\
\hline CENTRO DE DOCUMENTACIÓN EUSKERA & 0 & 0,00 & 1 & 0,59 \\
\hline CENTRO DE DOCUMENTACIÓN FARMACIA & 3 & 0,70 & 0 & 0,00 \\
\hline CENTRO DE DOCUMENTACIÓN GEOLOGÍA & 1 & 0,23 & 0 & 0,00 \\
\hline CENTRO DE DOCUMENTACIÓN HISTORIA & 2 & 0,46 & 0 & 0,00 \\
\hline CENTRO DE DOCUMENTACIÓN HUMANIDADES & 2 & 0,46 & 2 & 1,19 \\
\hline CENTRO DE DOCUMENTACIÓN INMOBILIARIAS & 1 & 0,23 & 0 & 0,00 \\
\hline CENTRO DE DOCUMENTACIÓN JUDICIAL & 1 & 0,23 & 0 & 0,00 \\
\hline CENTRO DE DOCUMENTACIÓN MEDICINA & 10 & 2,33 & 2 & 1,19 \\
\hline CENTRO DE DOCUMENTACIÓN MEDIOS AUDIOVISUALES & 3 & 0,70 & 3 & 1,78 \\
\hline CENTRO DE DOCUMENTACIÓN MUSICA & 2 & 0,46 & 0 & 0,00 \\
\hline CENTRO DE DOCUMENTACIÓN QUÍMICA & 1 & 0,23 & 0 & 0,00 \\
\hline CENTRO DOCUMENTACIÓN ADMINISTRACIÓN DE EMPRESAS & 1 & 0,23 & 0 & 0,00 \\
\hline CENTRO DOCUMENTACIÓN ARCHIVO HISTÓRICO & 1 & 0,23 & 1 & 0,59 \\
\hline CENTRO DOCUMENTACIÓN DERECHO & 11 & 2,57 & 0 & 0,00 \\
\hline CENTRO DOCUMENTACIÓN ECONÓMICAS & 2 & 0,46 & 2 & 1,19 \\
\hline EMPRESA ARQUITECTURA & 1 & 0,23 & 0 & 0,00 \\
\hline EMPRESA CONSULTORA & 49 & 11,47 & 13 & 7,73 \\
\hline EMPRESA EDITORIAL & 2 & 0,46 & 0 & 0,00 \\
\hline EMPRESA GESTIÓN DOCUMENTAL & 0 & 0,00 & 2 & 1,19 \\
\hline EMPRESA IFORMÁTICA & 13 & 3,04 & 5 & 2,97 \\
\hline
\end{tabular}




\begin{tabular}{|c|c|c|c|c|}
\hline EMPRESA INTERNET & 9 & 2,10 & 3 & 1,78 \\
\hline EMPRESA MULTIMEDIOS & 6 & 1,40 & 2 & 1,19 \\
\hline EMPRESA SERVICIOS DOCUMENTALES & 144 & 33,72 & 87 & 51,78 \\
\hline LIBRERÍA TOTALES & 2 & 0,46 & 0 & 0,00 \\
\hline \multicolumn{2}{|c|}{ T27 } & $100 \%$ & 168 & $100 \%$ \\
\hline
\end{tabular}

Tabla 2 - Análisis detallado de los Tipos de Organización que ofertan empleos.

\section{Conocimientos requeridos}

Los conocimientos requeridos para cubrir los puestos de trabajo presentan variaciones. Para los cargos de bibliotecario, sobre un total de 1511 condiciones solicitadas, el 44,34\% correspondió a conocimientos vinculados con el área de Tecnología de la Información (TI), el 29,11\% a Organización y Tratamiento de la Información (OTI), el $12,97 \%$ a Idiomas (IDM), 11,05 \% a Recursos y Servicios de Información (RSI), el 2,25 \% a Fundamentos de Biblioteconomía y Ciencias de la Información (FBCI), el 0,19\% a Gestión de Unidades de Información (GUI) y el $0,19 \%$ a Estadística (EST).

Los conocimientos más pedidos en el área de Tecnología de la Información (TI) se relacionan con bases de datos $(13,43 \%)$, búsqueda y recuperación de información en Internet (08,53 \%), conocimientos generales de informática $(04,50 \%)$, ABSYS $(04,30 \%)$ y programas de gestión automatizada de bibliotecas $(03,03)$. En el área Organización y Tratamiento de la Información (OTI) los conocimientos más solicitados se relacionan con catalogación (13,63\%), Formato Marc $(05,82 \%)$ y clasificación $(05,49 \%)$. Con respecto al área de Idiomas (IDM) los conocimientos más pedidos fueron el inglés (12,37\%), seguido por francés $(00,19 \%)$ y catalán $(00,19 \%)$.

En el caso de las ofertas para licenciados sobre un total de 662 condiciones requeridas, el $41,08 \%$ de los conocimientos demandados correspondió al área de Tecnología de la Información (TI), el 31,57\% a Organización y Tratamiento de la Información (OTI), el 13,74\% a Idiomas (IDM), el $08,76 \%$ a Recursos y Servicios de Información (RSI), el 00,60\% a Gestión de Unidades de Información (GUI), el 04,22\% a Fundamentos de Biblioteconomía y Ciencias de la Información (FBCl) y el 00,15\% a Estadística (EST).

Con respecto al área de Tecnología de la Información (TI) los conocimientos solicitados se relacionan con ABSYS $(08,61 \%)$, bases de datos $(08,15 \%)$, conocimientos de generales de informática $(05,13 \%)$, búsqueda y recuperación de información en Internet $(03,77 \%)$, digitalización $(03,47 \%)$ y programas de gestión automatizada de bibliotecas $(03,47 \%)$. Los conocimientos más requeridos en el área Organización y Tratamiento de la Información (OTI) fueron catalogación (11,93\%), Formato Marc (06,79\%), clasificación (04,38\%) y Formato IBERMARC $(04,22 \%)$. Con respecto al área de Idiomas (IDM) los conocimientos más demandados fueron el inglés (12,99\%). Finalmente en el área Recursos y Servicios de Información (RSI), la atención de usuarios $(03,32 \%$ ) y la gestión de documentos electrónicos $(01,05 \%)$ son los conocimientos más solicitados. En las tablas siguientes se presentan en detalles los diferentes tipos de conocimientos requeridos para cubrir los puestos de trabajo: 


\begin{tabular}{|l|c|c|c|c|}
\hline \multicolumn{1}{|c|}{ IWETEL } & \multicolumn{1}{c|}{ BIBLIOTECARIO } & \multicolumn{2}{c|}{ LICENCIADO } \\
\hline \multicolumn{1}{|c|}{ REQUISITOS / AREAS DE CONOCIMIENTO } & CANTIDAD & PORCENTAJE & CANTIDAD & PORCENTAJE \\
\hline TECNOLOGÍA DE LA INFORMACIÓN (TI) & 670 & 44,34 & 272 & 41,08 \\
\hline $\begin{array}{l}\text { ORGANIZACIÓN Y TRATAMIENTO DE LA INFORMACIÓN } \\
\text { (OTI) }\end{array}$ & 440 & 29,11 & 209 & 31,57 \\
\hline IDIOMAS (IDM) & 196 & 12,97 & 91 & 13,74 \\
\hline RECURSOS Y SERVICIOS DE INFORMACIÓN (RSI) & 167 & 11,05 & 58 & 08,76 \\
\hline GESTIÓN DE UNIDADES DE INFORMACIÓN (GUI) & 3 & 00,19 & 4 & 00,60 \\
\hline FUNDAMENTOS DE BIB. Y CIENCIAS DE LA INF. (FBCI) & 34 & 02,25 & 28 & 04,22 \\
\hline ESTADISTICA (EST) & 3 & 00,19 & 1 & 00,15 \\
\hline
\end{tabular}

Tabla 3 - Resumen de los conocimientos solicitados en las ofertas de empleos.

\begin{tabular}{|l|c|c|c|c|c|}
\hline \multicolumn{2}{|c|}{ IWETEL } & \multicolumn{2}{c|}{ BIBLIOTECARIO } & \multicolumn{2}{c|}{ LICENCIADO } \\
\hline REQUISITOS & AREA & CANTIDAD & PORCENTAJE & CANTIDAD & PORCENTAJE \\
\hline ESTADÍSTICA & EST & 3 & 0,19 & 1 & 0,15 \\
\hline ARCHIVISTICA & FBCI & 21 & 1,38 & 20 & 3,02 \\
\hline RESTAURACIÓN & FBCI & 3 & 0,19 & 0 & 0,00 \\
\hline NORMAS ISAD (G) & FBCI & 10 & 0,66 & 8 & 1,20 \\
\hline PLANEAMIENTO / GESTIÓN & GUI & 3 & 0,19 & 4 & 0,60 \\
\hline INGLES & IDM & 187 & 12,37 & 86 & 12,99 \\
\hline CATALAN & IDM & 3 & 0,19 & 1 & 0,15 \\
\hline EUSKERA & IDM & 2 & 0,06 & 1 & 0,15 \\
\hline FRANCES & IDM & 3 & 0,19 & 1 & 0,15 \\
\hline ALEMAN & IDM & 1 & 0,06 & 1 & 0,15 \\
\hline PORTUGUÉS & IDM & 0 & 0,00 & 1 & 0,15 \\
\hline CATALOGACIÓN & OTI & 206 & 13,63 & 79 & 11,93 \\
\hline CLASIFICACIÓN & OTI & 83 & 5,49 & 29 & 4,38 \\
\hline FORMATO IBERMARC & OTI & 19 & 1,25 & 28 & 4,22 \\
\hline PROCESOS TECNICOS & OTI & 0 & 0,00 & 1 & 0,15 \\
\hline ORGANIZACIÓN CONOCIMIENTO & OTI & 1 & 0,06 & 0 & 0,00 \\
\hline CATMARC & OTI & 15 & 0,99 & 11 & 1,66 \\
\hline FORMATO MARC & OTI & 88 & 5,82 & 45 & 6,79 \\
\hline
\end{tabular}




\begin{tabular}{|c|c|c|c|c|c|}
\hline INDIZACIÓN - RESUMEN & OTI & 20 & 1,32 & 10 & 1,51 \\
\hline DUBLIN CORE & OTI & 1 & 0,06 & 1 & 0,15 \\
\hline Z3950 & OTI & 1 & 0,06 & 1 & 0,15 \\
\hline TESAURUS & OTI & 1 & 0,06 & 0 & 0,00 \\
\hline METADATOS & OTI & 3 & 0,19 & 1 & 0,15 \\
\hline FORMATO MARC 21 & OTI & 0 & 0,00 & 1 & 0,15 \\
\hline GESTION DOCUMENTAL & $\mathrm{RSI}$ & 34 & 2,25 & 16 & 2,41 \\
\hline GESTIÓN INFORMACIÓN & RSI & 18 & 1,19 & 3 & 0,45 \\
\hline GESTIÓN CONOCIMIENTO & $\mathrm{RSI}$ & 11 & 0,72 & 7 & 1,05 \\
\hline REFERENCIA & RSI & 48 & 3,17 & 2 & 0,30 \\
\hline ATENCIÓN USUARIOS & RSI & 33 & 2,18 & 22 & 3,32 \\
\hline GESTION DOCUMENTOS ELECTRÓNICOS & $\mathrm{RSI}$ & 13 & 0,86 & 7 & 1,05 \\
\hline DESARROLLOS DE COLECCIONES & $\mathrm{RSI}$ & 2 & 0,13 & 0 & 0,00 \\
\hline ALFABETIZACIÓN INFORMACIONAL & RSI & 2 & 0,13 & 0 & 0,00 \\
\hline ANIMACIÓN A LA LECTURA & RSI & 3 & 0,19 & 0 & 0,00 \\
\hline BIBLIOMETRIA & RSI & 1 & 0,06 & 1 & 0,15 \\
\hline FORMACIÓN DE USUARIOS & $\mathrm{RSI}$ & 2 & 0,13 & 0 & 0,00 \\
\hline BASE DE DATOS & $\mathrm{TI}$ & 203 & 13,43 & 54 & 8,15 \\
\hline $\begin{array}{l}\text { INTERNET (BÚSQUEDA Y } \\
\text { RECUPERACIÓN DE INFO) }\end{array}$ & $\mathrm{TI}$ & 129 & 8,53 & 25 & 3,77 \\
\hline INFORMÁTICA (OFFICE) & $\mathrm{TI}$ & 68 & 4,50 & 34 & 5,13 \\
\hline DIGITALIZACIÓN & $\mathrm{TI}$ & 39 & 2,58 & 23 & 3,47 \\
\hline PROGRAMA ABSYS & $\mathrm{TI}$ & 65 & 4,30 & 57 & 8,61 \\
\hline $\begin{array}{l}\text { PROGRAMACIÓN WEB (HTML JAVA SQL, } \\
\text { PHP) }\end{array}$ & $\mathrm{TI}$ & 18 & 1,19 & 11 & 1,66 \\
\hline $\begin{array}{l}\text { PROGRAMA AUTOMATIZACIÓN } \\
\text { BIBLIOTECAS }\end{array}$ & $\mathrm{TI}$ & 58 & 3,83 & 23 & 3,47 \\
\hline INFORMATICA DOCUMENTAL & $\mathrm{TI}$ & 4 & 0,26 & 0 & 0,00 \\
\hline PROGRAMAS GESTION DOCUMENTAL & $\mathrm{TI}$ & 6 & 0,39 & 1 & 0,15 \\
\hline $\begin{array}{l}\text { DISENO WEB (COREL DREAMWEAVER, } \\
\text { PHOTOSHOP) }\end{array}$ & $\mathrm{TI}$ & 22 & 1,44 & 11 & 1,66 \\
\hline BIBLIO 3000 & $\mathrm{TI}$ & 1 & 0,06 & 0 & 0,00 \\
\hline PROGRAMAS VISUAL BASIC & $\mathrm{TI}$ & 2 & 0,13 & 0 & 0,00 \\
\hline VTLS & $\mathrm{TI}$ & 28 & 1,85 & 19 & 2,87 \\
\hline REDES & $\mathrm{TI}$ & 1 & 0,06 & 2 & 0,30 \\
\hline LOTUS & $\mathrm{TI}$ & 2 & 0,13 & 0 & 0,00 \\
\hline
\end{tabular}




\begin{tabular}{|l|c|c|c|c|c|}
\hline EASYCAT / EASY POC & TI & 4 & 0,26 & 0 & 0,00 \\
\hline PHP & TI & 2 & 0,13 & 1 & 0,15 \\
\hline PROGRAMA SABINI & TI & 2 & 0,13 & 0 & 0,00 \\
\hline PROGRAMAS CMS (OPEN SOURCE) & TI & 1 & 0,06 & 1 & 0,15 \\
\hline PROGRAMA DOMUS & TI & 0 & 0,00 & 0 & 0,00 \\
\hline PROGRAMA AMICUS & TI & 2 & 0,13 & 0 & 0,00 \\
\hline PROGRAMA INNOPAC & TI & 1 & 0,06 & 0 & 0,00 \\
\hline TECNOLOGÍA DE LA INFORMACIÓN & TI & 1 & 0,06 & 0 & 0,00 \\
\hline PROGRAMA DOCUMENTATUM & TI & 1 & 0,06 & 0 & 0,00 \\
\hline BIB. VIRTUAL & TI & 1 & 0,06 & 0 & 0,00 \\
\hline PROGRAMA INMAGIC & TI & 0 & 0,00 & 2 & 0,30 \\
\hline PROGRAMA DBTEXT & TI & 0 & 0,00 & 1 & 0,15 \\
\hline LINUX & TI & 3 & 0,19 & 5 & 0,75 \\
\hline PROGRAMAS GESTION DOCUMENTAL & TI & 5 & 0,33 & 0 & 0,00 \\
\hline WINISIS & TI & 1 & 0,06 & 1 & 0,15 \\
\hline CATALIS & TI & 0 & 0,00 & 1 & 0,15 \\
\hline & & 1511 & 100 & 662 & 100 \\
\hline
\end{tabular}

Tabla 4 - Análisis detallado de los conocimientos solicitados en las ofertas de empleos.

\section{Ámbito de las organizaciones y modalidades de los contratos}

Con respecto a tipo de la organización (pública o privada) que ofrece empleos, los resultados presentan diferencias. En las ofertas de empleo publicadas aparece una preponderancia de ofrecimientos provenientes del ámbito privado. En efecto para puesto de bibliotecario el $72,59 \%$ se origina en el ámbito privado, y el $27,40 \%$ restante en el ámbito público. Esta tendencia se acrecienta en las ofertas publicadas para licenciados en donde el $82,73 \%$ proviene del ámbito privado y solo el $17,26 \%$ de las ofertas se originan en el ámbito estatal. En la siguiente tabla se presentan los resultados obtenidos:

\begin{tabular}{|l|c|c|c|c|}
\hline IWETEL 2000-2007 & \multicolumn{2}{|c|}{ BIBLIOTECARIO } & \multicolumn{2}{c|}{ LICENCIADO } \\
\hline TIPO DE AMBITO & CANTIDAD & PORCENTAJE & CANTIDAD & PORCENTAJE \\
\hline PRIVADO & 310 & 72,59 & 139 & 82,73 \\
\hline PUBLICO & 117 & 27,40 & 29 & 17,26 \\
\hline \multicolumn{1}{|c|}{ TOTALES } & 427 & $100 \%$ & 168 & $100 \%$ \\
\hline
\end{tabular}

Tabla 5 - Ámbito de las Organizaciones que realizan ofertas. 


\section{Modalidad de los contratos (temporario o permanente)}

En relación a la modalidad de los contratos (temporario o permanente) también encontramos diferencias. En ofertas para bibliotecarios el $82,90 \%$ correspondió a contratos temporarios y el 17,09\% restante a contratos permanentes. Con respecto a los trabajos para licenciados esta tendencia se incrementa. El 89,88\% de las ofertas publicadas se relaciona con puestos de carácter temporario y solo el 10,11\% de los trabajos publicados eran de carácter permanente. En la tabla № 6 se presentan los resultados obtenidos:

\begin{tabular}{|l|c|c|c|c|}
\hline IWETEL 2000-2007 & \multicolumn{2}{|c|}{ BIBLIOTECARIO } & \multicolumn{2}{c|}{ LICENCIADO } \\
\hline TIPO DE CONTRATO & CANTIDAD & PORCENTAJE & CANTIDAD & PORCENTAJE \\
\hline TEMPORARIO & 354 & 82,90 & 151 & 89,88 \\
\hline PERMANENTE & 73 & 17,09 & 17 & 10,11 \\
\hline \multicolumn{1}{|c|}{ TOTALES } & 427 & $100 \%$ & 168 & $100 \%$ \\
\hline
\end{tabular}

Tabla 6 - Tipos de contratos ofertados.

\section{Conclusiones}

Al analizar los resultados de las ofertas hemos comprobado que los puestos ofrecidos mayoritariamente provienen de organizaciones empresariales, en su mayoría de empresas de servicios documentales y de consultoría. Con respecto al ámbito al que pertenecen las organizaciones (público o privado), observamos que las ofertas publicadas pertenecen mayoritariamente al ámbito privado.

Estos resultados difieren con los datos obtenidos en los estudios realizados previamente por Espelt y Pons (1993), Moreiro, Moscoso y Ortiz-Repiso (1995), Camps y Crespán (1996), Muñoz Escolá (1997), Moreiro y Caridad (1998), Galán Cruzado (1999), Alonso y Vázquez (2000), y Frías y otros autores (2000). En estas investigaciones los resultados indican que los trabajos ofertados provienen mayormente de bibliotecas y centros de documentación y que el ámbito de las organizaciones es predominantemente estatal. En este punto notamos un cambio importante en los últimos años en relación a la demanda de profesionales por parte de las organizaciones empresariales.

Con respecto al tipos de contratos existe un predomino de contratos temporarios. En este punto defiere el estudio previo realizado por Universidad de Barcelona (2003) donde las ofertas de empleo son mayormente de contratos permanentes.

En relación a lo conocimientos requeridos se observa que tanto en las ofertas para bibliotecario como para licenciado se solicitan mayormente conocimientos en el área de Tecnología de la Información (TI), en Organización y Tratamiento de la Información (OTI), y en Idiomas (IDM). Estos resultados coinciden con los datos obtenidos por Sanz-Casado, Martin-Moreno, García-Zorita y Lascurian-Sanchez (2002).

Los cambios sociales, económicos, culturales y tecnológicos presentes en los últimos años, han generado que el ejercicio profesional de la biblioteconomía y la documentación, se encuentre inmerso en un proceso de evolución. La sociedad cambia y las nuevas demandas y problemáticas sociales, requieren que la profesión se transforme. En este contexto surge la necesidad de analizar las necesidades profesionales demandadas por la sociedad. En este sentido consideramos prioritario que en futuras investigaciones se amplíen los estudios de las demandas laborales a otras listas de interés de la especialidad y a otras fuentes de información similares que se encuentran presentes en el contexto iberoamericano.

Finalmente debido a que en las ofertas laborales la mayor demanda de conocimientos solicitados se relacionan con Tecnología de la Información, Organización y Tratamiento de la Información e Idiomas, estimamos recomendable que las Escuelas de Bibliotecología y Ciencias de la Información consideren y prioricen estos contenidos en los diseños curriculares de sus carreras. 


\section{Bibliografía}

Alonso Arévalo, J. y Vázquez Vázquez, M. (2000). Características del comportamiento del mercado de trabajo en Biblioteconomía, Archivística y Documentación: bienio 98/99. Anales de Documentación, vol. 3, 9-24.

Camps, E. y Crespán, J. L. (1996). Navegar en un entorn turbulent. Els bibliotecaris-documentalistes a Catalunya: situació actual i perspectives de futur". Item. Revista de Biblioteconomía i Documentació, vol.18, 83-101.

Espelt, C. y Pons, A. (1993). Dades sobre la incorporació laboral de les darreres promocions de diplomats de l'Escola Jordi Rubió i Balaguer". Item, vol. 13, 95-103

Frías Montoya, J. A., et al. Formación e inserción laboral de los titulados universitarios en Biblioteconomía y Documentación por la Universidad de Salamanca. (2000). V Encuentro de la Asociación de Educación e Investigación en Bibliotecología, Archivología, Ciencias de la Información y Documentación de Iberoamérica y El Caribe, p. 475-492, Granada, España.

Galán Cruzado, F. (1999). Nuevos profesionales en la administración: una visión de la oferta de empleo público para información y documentación. II Jornadas Andaluzas de Documentación. Nuevos mercados, nuevos usuarios, p. 119-137. Granada, España: Asociación Andaluza de Documentalistas.

Moreiro González, J. A. y Caridad, M. (1998). Acerca de los métodos de estudio de la relación entre las condiciones laborales y formativas en biblioteconomía y documentación: el caso de la Universidad Carlos III. Anales de Documentación, vol.1, 137-153.

Muñoz Escolá, M. M. (1997) Análisis de la situación laboral y de formación de los estudiantes y diplomados en Biblioteconomía y Documentación por la Universidad de Zaragoza. Revista General de Información y Documentación, vol.7 (1), 324-344.

Sanz-Casado, E; Martin-Moreno, C; Garcia-Zorita, C y Lascurian-Sanchez M L. (2002). Como responden los estudios de bibliotecología de las universidades españolas a las nuevas demandas sociales?. Ciencia da Informacao, vol. 31 (2) 21-29.

Universitat de Barcelona. Facultat de Biblioteconomia i Documentació, Diplomatura en Biblioteconomia i Documentació. (2003). Informe d'autoavaluació, versió definitiva que incorpora els comentaris rebuts de la comunitat universitaria, p. 18-19.Barcelona, España: Universitat de Barcelona, Facultat de Biblioteconomia i Documentació.

\section{Datos del autor}

\section{Carlos Hugo Artaza}

Lic. en Bibliotecología y Documentación. Especialista y Magister en Docencia Universitaria. (Universidad Nacional de Mar del Plata, Argentina) Doctorando en Información y Documentación Científica (Universidad de Granada, España). Docente del Departamento de Documentación. Facultad de Humanidades. UNMDP.

cartaza@mdp.edu.ar

Recibido - Received: 2011-08-05

Aceptado - Accepted: 2011-09-30

\section{(cc) BY-NC-ND}

This work is licensed under a Creative Commons Attribution-Noncommercial-No Derivative Works 3.0 United States License.

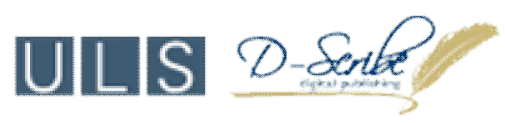

This journal is published by the University Library System of the University of Pittsburgh as part of its $\underline{D-S c r i b e}$ Digital Publishing Program and is cosponsored by the University of Pittsburgh Press. 